\title{
论文
}

\section{基于GPS测站垂向速度的南极地区冰川均衡调整 (GIA)模型分析}

\author{
李斐, 马超, 张胜凯”, 雷锦舀, 郝卫峰, 张卿川, 李文浩 \\ 武汉大学中国南极测绘研究中心, 武汉 430079 \\ * 通讯作者, E-mail: zskai@whu.edu.cn
}

收稿日期：2019-03-27; 收修改稿日期：2019-09-11; 接受日期：2019-10-22; 网络版发表日期：2020-01-02 国家重点研发计划项目(编号：2017YFA0603104)、国家自然科学基金重点项目(批准号：41531069)和武汉大学自主科研学科交叉类项目(编 号: 2042017kf0209)资助

\begin{abstract}
摘要由于数据稀缺, 南极冰川均衡调整(glacial isostatic adjustment, GIA)建模相比北美和北欧古冰盖区域更为困 难, 现有GIA 模型在南极地区还存在较大的不确定性. 空间大地测量技术不断发展和进步, 为GIA建模提供了外部 检核和新的约束。利用GAMIT/GLOBK10.5软件对南极及周边区域73 个测站1996 2014年的GPS数据进行解算和 平差，并考虑有色噪声估计其地壳垂向运动速度。为了研究不同因素对地壳垂向运动的影响，将GPS测站垂向速 度与Argus等(2014)、Thomas等(2011)的GPS结果以及各GIA模型预测结果划分区域进行比较, 主要包括南极半岛 北部、龙尼-菲尔希纳冰架、阿蒙森海沿岸、罗斯冰架、埃里伯斯火山、西南极内陆、东南极沿海等七个区域, 结果表明在南极半岛北部和阿蒙森海沿岸派恩艾兰湾地区存在现今冰雪负荷变化引起的显著地壳弹性抬升, 在 埃尔伯斯火山区域存在的显著下沉可能与其地下岩浆活动有关, 东南极沿海测站垂向运动速度都不显著, 其他区 域的测站垂向运动主要受到GIA的影响. 对文章GPS结果与各GIA模型的相关性和GIA模型的不确定性进行分析, 结果表明ICE-6G(VM5a)模型与文章GPS结果的一致性最好，其次是Geruo13模型，而W12a和IJ05_R2系列模型与 本文GPS结果一致性相对较差。虽然目前南极GIA模型相比以前版本的模型有了很大改进，但是南极地区GIA建 模的状况仍然不容乐观: GIA模型相比北美地区不确定性偏大, 在BENN等部分测站上存在较大不符值, 在现今冰 雪负荷变化显著地区由于弹性改正模型不确定性偏大而缺乏精确GPS约束.
\end{abstract}

关键词南极, GPS, 冰川均衡调整, 垂向速度, 不确定性

\section{1 引言}

通常认为南极的长期运动主要包括水平刚性板块 旋转加上由过去和现今冰-海水负荷变化引起的三维
形变(King和Santamaría-Gómez, 2016), 后者统称为冰 川均衡调整(glacial isostatic adjustment, GIA). 由于大 多数GIA模型主要考虑的是末次冰盛期的冰量变化对 现今的影响, 而并没有考虑最近 2000 年的冰量变化 
(Nield等, 2015), 因此本文研究的GIA是指黏弹地球对 末次冰期(21 6ka)地表冰和海水负荷改变的响应，也 称冰后回弹. 末次冰盛期(last glacial maximum, LGM) 在约 $20 \mathrm{ka}$ 结束, 那时包括北美、格陵兰岛、芬诺斯坎 底亚和南极在内的冰盖开始融化, 在约 $6 \mathrm{ka}$ 大部分融化 已经结束, 全球平均海平面因此上升了120 130m(Peltier, 2004). 冰、海洋质量的重新分布意味着地球表面 主要负荷的重新分配, 这些负荷改变了地球的重力 场、旋转极和速度, 并产生黏弹性响应, 其中包括固 体地球的表面形变(Mitrovica等, 2009), 冰川地质学和 古海平面数据可以提供对这种形变和质量重分布历史 的约束. GIA模型能够对板块运动、地壳垂向运动、 大地水准面变化、海平面变化、GRACE重力场、卫 星测高和全球气候研究等提供重要改正(汪汉胜等, 2009; Ivins等, 2013; Prandi等, 2009), 还可以辅助GPS 和VLBI数据的分析, 建立更稳健的国际地球参考框架 (Altamimi和Collilieux, 2009), 提供地形地貌包括海陆 分布和反射率的边界条件(Whitehouse等, 2012a).

通过对目前几种GIA模型进行比较, 发现模型之 间存在着较大的差异，如在南极冰盖区域不同模型之 间的垂向地表位移差别达到几个 $\mathrm{mm} \mathrm{a}^{-1}$, 说明GIA建 模存在较大的不确定性，原因是目前还没有足够的数 据用于约束. GIA模型的不确定性主要来自两个方面: 一是冰模型中冰盖厚度历史不够精确; 二是地球模型 中有相当大的简化. 冰后回弹模型包含三个高度相关 的参数, 作为位置和时间函数的冰盖厚度 (冰消历 史)、作为深度函数的位于岩石圈下方的地幔黏滞度 和弹性岩石圈的厚度，冰消历史、地幔黏滞度剖面或 岩石圈厚度中的误差可能会影响另外两个参数的估 计. 另外地震数据表明, 在南极洲的地幔中有较大的黏 滞度变化, 这种变化会影响GIA模型的预测, 然而大多 数的GIA模型都假定为均匀的径向分层地幔黏滞度, 没有进行黏弹性结构的横向非均匀性假设也可能引入 额外的不确定性(Wang 和Wu, 2006; Wu和Wang, 2006; Wang等, 2008).

地幔黏滞度对固体地球调整的响应时间起决定作 用, 由于地幔黏滞度足够高使得该形变至今仍然可以 测量, 其中地球表面位移的大地测量观测值和卫星、 地面重力场观测值的变化最为显著. 因此, 对这些参 数的测量可以深入了解过去的冰盖结构和海平面变 化，以及地球结构和流变学(Milne等，2001；King等,
2010). 利用连续GPS可以获得高精度的地壳运动速度, 为GIA建模提供了外部检核和新的约束. 南极IGS连续 跟踪站大都依托于各国相应的科学考察站，基本上都 分布在南极沿海地区. 国际南极研究科学委员会 (Scientific Committee for Antarctic Research, SCAR)从 20 世纪90年代初开始, 先后建立了WAGN、TAMDEF、VLNDEF以及其他GPS会战网络. 2007/2008年 国际极地年间, 由多国合作开始在南极实施POLENET (The Polar Earth Observing Network)计划, 该计划的主 要任务是在极地布设GPS 与地震等应用于地球科学研 究的常年连续观测仪器(鄂栋臣和张胜凯, 2006). 近年 来, 随着POLENET计划的实施和南极IGS测站的不断 扩充, 南极连续GPS测站无论数量还是分布上都有了 较大改善, 可获得更长时间序列和更高观测精度的 GPS数据, 用于检验和约束GIA模型.

\section{GPS数据处理}

\subsection{GPS数据来源}

要准确估计GPS跟踪站的速度和精度, 通常要求 坐标序列的时间跨度大于 2.5 年(Blewitt和Lavallée, 2003). 本文选取南极地区 $\left(60^{\circ} \mathrm{S}\right.$ 以南 $) 65$ 个 GPS站点及 其周边区域 $\left(60^{\circ} \mathrm{S}\right.$ 以北 $) 8$ 个 GPS站点, 测站分布如图 1 所示, 其中IGS站点有 12 个、POLENET站点39个、南 极埃里伯斯火山(Antarctica Erebus, AE)附近的6个站 点、中国南极中山站(ZHON) 以及其他组织和机构的7 个站点, 数据跨度为1996 2014年, 测站最短时间跨度 大于 2.5 年, 最长时间跨度为 19 年.

\section{2 基线解算}

GPS数据的基线解算采用GAMIT/GLOBK10.5 高 精度数据处理软件. 由于要解算的测站数多达 73 个, 全网解算效率很低. 姜卫平等(2011)认为虽然从理论 上看来划分子网会影响数学模型的严密性, 但在实际 计算时这种影响很小, 与全网处理相比精度相当. 斯 克里普斯海洋学研究所(Scripps Institute of Oceanography, SIO)在处理全球GPS网时, 也将全球网划分为 多个子网, 先在各子网内进行基线处理, 再联合各子 网进行联合平差. 本文在进行解算时划分了三个子网, 子网间具有至少 6 个公共站点, 最后统一进行平差. GAMIT基线解算策略及参考准则如下: (1) 解算类型 


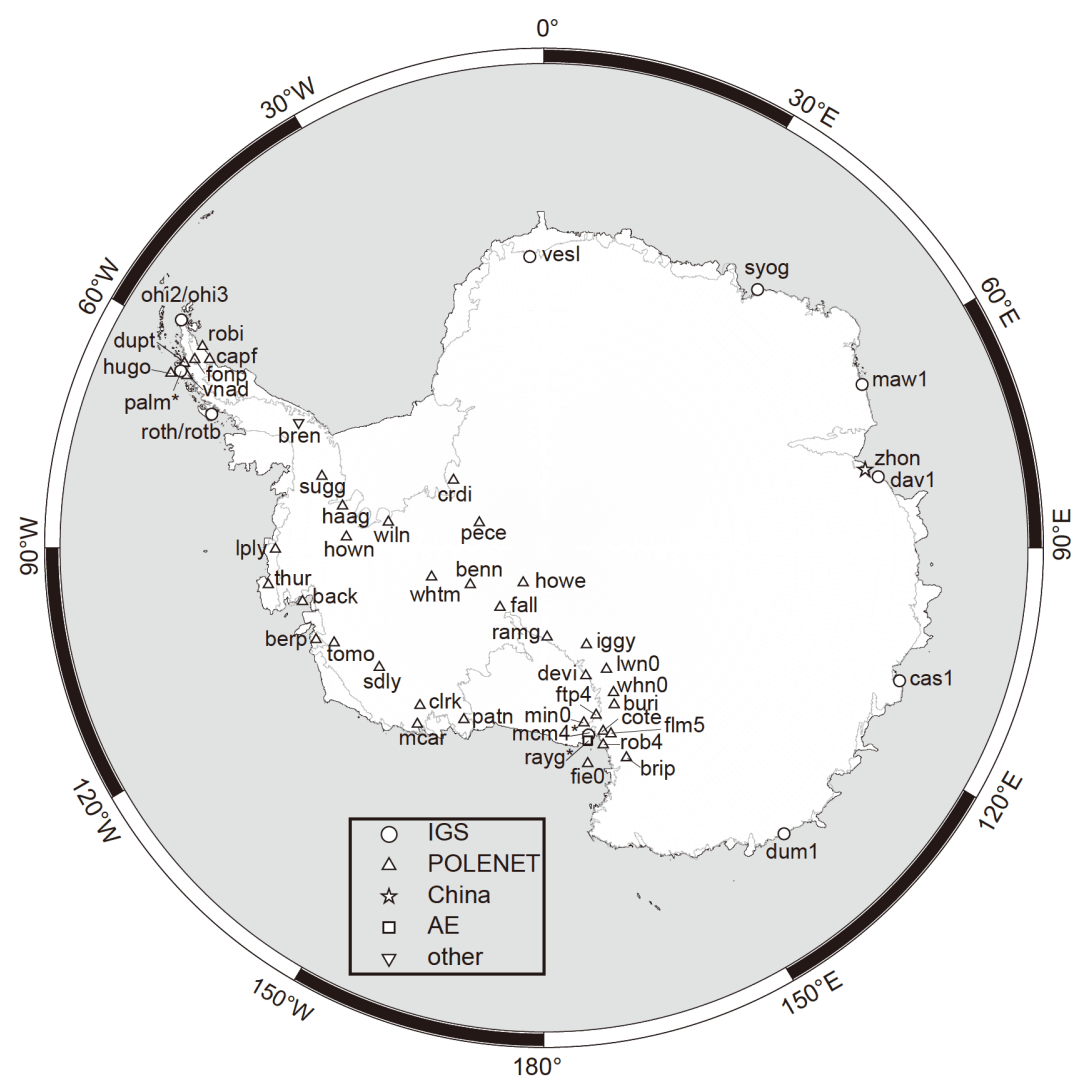

图 1 南极地区 $\left(60^{\circ} \mathrm{S}\right.$ 以南 $) 65$ 个连续 GPS站点分布图

圆圈代表IGS的测站, 三角形代表POLENET的测站, 五角星代表中山站(ZHON), 矩形代表南极埃里伯斯火山GPS网(Antarctica Erebus, AE)的

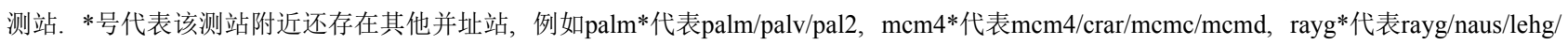
elg2/cong/hooz/macg

为松弛解 (RELAX), 同时解算卫星轨道、测站坐标; (2) 观测值使用无电离层组合、自动修复周跳模式 (LC_AUTCLN); (3) IGS站先验坐标约束为 $0.05 、 0.05$ 和 $0.10 \mathrm{~m}$, 其他站先验坐标约束为 $100 、 100$ 和 $100 \mathrm{~m}$; (4) 截止高度角为 $15^{\circ}$; (5) 历元间隔为 $30 \mathrm{~s}$; (6) 对流层延迟 改正采用Saastamoinen模型; (7) 潮汐改正采用 FES2004模型; (8) 光压摄动改正采用BERNE模型; (9) 参考框架为ITRF 2008; (10) 其他均采用GAMIT/ GLOBK10.5默认设置(马超等, 2016).

\section{3 网平差}

基线处理完成后，使用GLOBK软件将基线解在 ITRF2008框架下进行网平差. 由于本文解算的是区域 GPS网络, 为了维持框架稳定, 本文加入了全球IGS网 络1996 2014年igs1 igs9共计9个子网的基线解算结果 (由SOPAC提供下载, 链接为ftp://garner.ucsd.edu/ar- chive/garner/solutions/global/), 与IGS全球网基线解算 结果进行联合平差. 选用IGS重处理结果(IGb08)中91 个核心站作为框架稳定站, 对稳定站坐标N、E、U方 向分别约束为 $0.01 、 0.01 、 0.05 \mathrm{~m} ; \mathrm{UT} 1$ 及误差约束为 $0.25 、 0.1 \mathrm{~s}$, 极点水平坐标和误差约束为 $0.25 、 0.25$ 、 $0.1 、 0.1 \mathrm{~m}$. 平差后各测站坐标时间序列水平分量的重 复性(加权均方根误差)均优于 $5 \mathrm{~mm}$, 垂直分量的重复 性均优于 $10 \mathrm{~mm}$ (李斐等, 2016).

\section{4 异常值剔除}

GLOBK网平差得到的时间序列中存在少数异常 值, 可能是由于外界环境变化或仪器内部元件的变化 等因素引起, 会对时间序列分析造成偏差, 所以有必 要进行剔除. 本文使用三倍四分位数差法则(IQR, Interquartile Range)(Nikolaidis, 2002)对坐标时间序列中 的异常值进行剔除, 剔除标准为 
$\left|\widehat{x}_{i}-\operatorname{median}\left(x_{i-w / 2, i+w / 2}\right)\right|>3 \cdot \operatorname{IDR}\left(x_{i-w / 2, i+w / 2}\right)$,

式中, 被探测值 $\widehat{x}_{i}$ 代表第 $i$ 个历元的坐标值, $\operatorname{median}\left(x_{i-w / 2, i+w / 2}\right)$ 代表以该历元为中心、窗口宽度为 $w$ 范围内的中位数, $\operatorname{IDR}\left(x_{i-w / 2, i+w / 2}\right)$ 代表窗口内坐标的 四分位数差, 即先将窗口内的时间序列按从小到大排 列, 然后取第 $3 / 4$ 和第 $1 / 4$ 个数值之差, 得到IQR 值. 本文 在剔除异常值时取窗口宽度为 1 年.

\section{5 阶跃改正}

部分GPS测站时间序列由于地震或天线变动发生 阶跃，本文在平差时使用GAMIT/GLOBK10.5 提供的 地震重命名文件和阶跃前后坐标得到阶跃历元和大 小, 并对其进行改正. 经过上述改正后, 测站WHTM仍 然存在阶跃，本文对其阶跃前后的坐标时间序列使用 CATS软件进行分段拟合, 求出阶跃前后的坐标拟合差 (即阶跃值), 然后对阶跃后的坐标进行改正. 测站 WHTM阶跃改正前、后坐标时间序列如图2所示，可 以看出在历元2011.978WTHM的U方向坐标时间序列 存在一个 $30 \mathrm{~mm}$ 的阶跃, 在阶跃改正后该测站的垂向速 度和误差估计都有明显减小，速度从13.65下降到 $4.38 \mathrm{~mm} \mathrm{a}^{-1}$, 误差从 3.66 减小到 $1.66 \mathrm{~mm} \mathrm{a}^{-1}$.
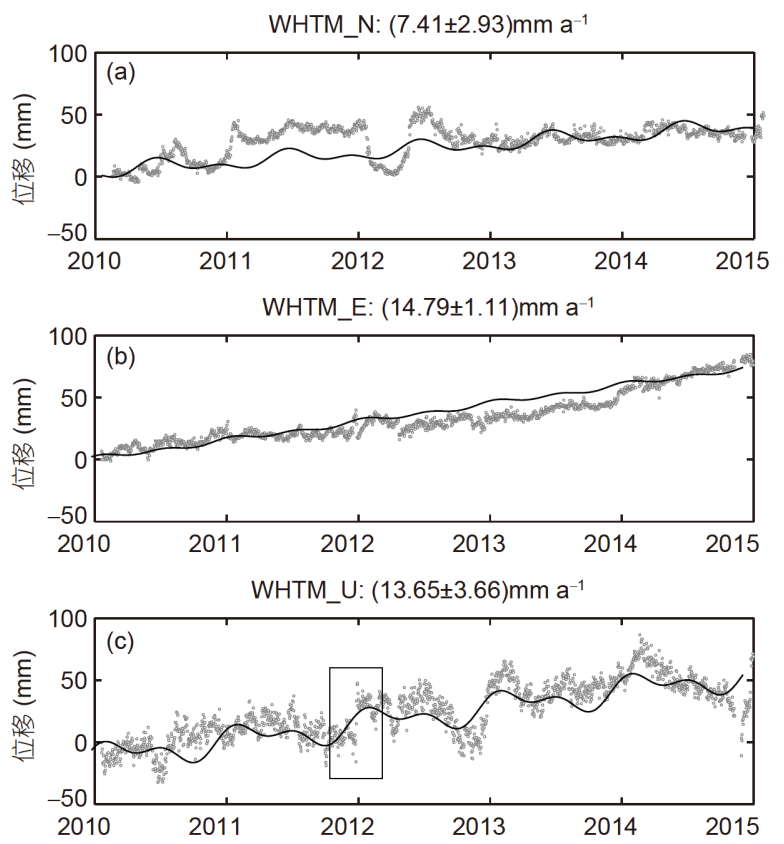

\section{6 测站速度估计与并址站合并}

连续GPS站坐标时间序列中不仅存在白噪声, 还 存在有色噪声, 例如闪炼噪声和随机游走噪声等, 这 些噪声对GPS时间序列分析有重要影响(姜卫平和周 晓慧，2014)。李伟伟等(2014)通过分析南极大陆12个 IGS站的坐标时间序列, 发现南极区域最优噪声模型 为白噪声+闪烁噪声+随机漫步噪声. 因此本文使用最 大似然估计法考虑白噪声、闪炼噪声和随机漫步噪 声，分别估计了南极区域73个测站N、E、U方向的地 壳运动速度. 由于部分测站之间距离很近 ( $5 \mathrm{~km}$ 以内), 可以看作是并址站，但各并址站速度并不一致，需要 进行合并. 本文中并址站一共有四组，如表1所示，以 各测站速度中误差的倒数为权, 计算各并址站速度的 加权平均值来表示它们的平均速度. 为了与合并前的 测站进行区别便于以后使用，本文将速度合并后的并 址站来分别命名为 $\mathrm{MCM} 4 *$ 、 PALM* $* \mathrm{OHI} 2 *$ 和 $\mathrm{ROTH}^{*}$. 合并之后总测站共 66 个，其中南极大陆上有 58 个, 南极大陆以外有 8 个, 由于本文的研究重点是南 极区域, 接下来将重点分析南极大陆的GPS测站速度.

\section{7 参考框架转换的影响}

为了对GPS测站垂向速度和GIA模型预测进行准
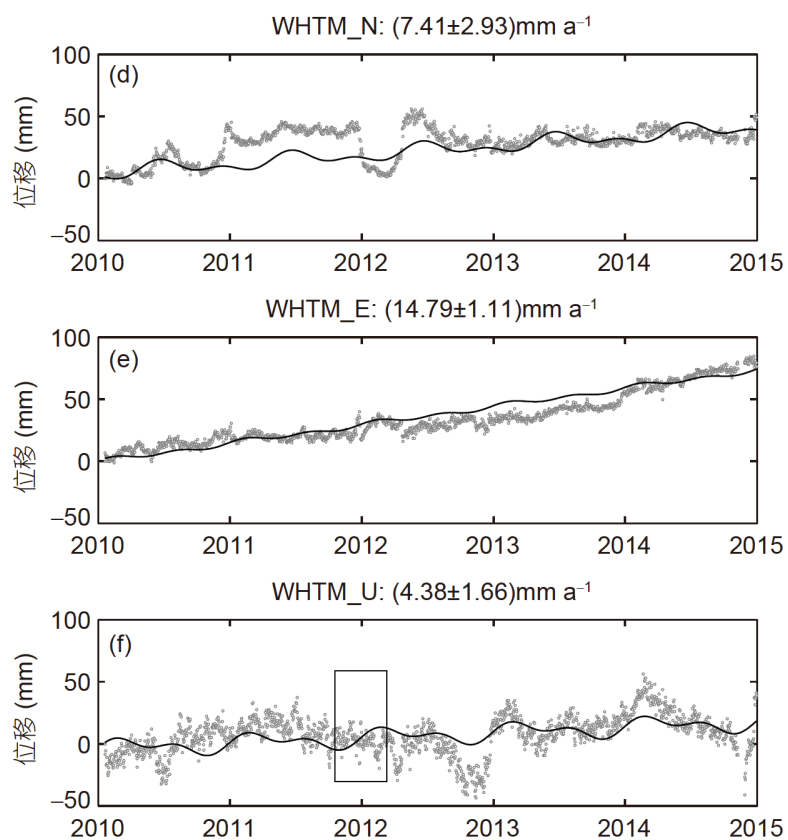

图 2 测站阶跃改正前((a) (c))、改正后((d) (f))坐标时间序列比较 灰色点为测站时间序列, 黑色实线代表线性周期项拟合结果, 方框处为主要阶跃改正 
表 1 并址站垂向速度合并

\begin{tabular}{|c|c|c|c|c|c|}
\hline 测站 & 经度 & 纬度 & $V_{\mathrm{u}}\left(\mathrm{mm} \mathrm{a}^{-1}\right)$ & $\Delta V_{\mathrm{u}}\left(\mathrm{mm} \mathrm{a}^{-1}\right)$ & 合并后 $V_{\mathrm{u}}\left(\mathrm{mm} \mathrm{a}^{-1}\right)$ \\
\hline CRAR & $166.67^{\circ} \mathrm{E}$ & $77.84^{\circ} \mathrm{S}$ & 0.66 & 0.34 & \multirow{4}{*}{0.69} \\
\hline MCM4 & $166.67^{\circ} \mathrm{E}$ & $77.84^{\circ} \mathrm{S}$ & 0.62 & 0.26 & \\
\hline MCMC & $166.67^{\circ} \mathrm{E}$ & $77.84^{\circ} \mathrm{S}$ & 0.99 & 0.56 & \\
\hline MCMD & $166.67^{\circ} \mathrm{E}$ & $77.84^{\circ} \mathrm{S}$ & 0.77 & 0.56 & \\
\hline PAL2 & $64.05^{\circ} \mathrm{W}$ & $64.78^{\circ} \mathrm{S}$ & 6.31 & 0.64 & \multirow{3}{*}{6.18} \\
\hline PALM & $64.05^{\circ} \mathrm{W}$ & $64.78^{\circ} \mathrm{S}$ & 6.08 & 0.33 & \\
\hline PALV & $64.05^{\circ} \mathrm{W}$ & $64.78^{\circ} \mathrm{S}$ & 6.28 & 1.11 & \\
\hline OHI2 & $57.90^{\circ} \mathrm{W}$ & $63.32^{\circ} \mathrm{S}$ & 3.17 & 1.14 & \multirow{2}{*}{3.15} \\
\hline OHI3 & $57.90^{\circ} \mathrm{W}$ & $63.32^{\circ} \mathrm{S}$ & 3.09 & 3.26 & \\
\hline ROTB & $68.13^{\circ} \mathrm{W}$ & $67.57^{\circ} \mathrm{S}$ & 3.46 & 0.97 & \multirow{2}{*}{4.11} \\
\hline ROTH & $68.13^{\circ} \mathrm{W}$ & $67.57^{\circ} \mathrm{S}$ & 5.45 & 2.00 & \\
\hline
\end{tabular}

确的比较和分析, 必须保证两者所在参考框架相同或 者具有一致性. GPS垂向速度是在ITRF2008参考框架 下估计的，该框架是以整个地球系统的质心 $(\mathrm{CM})$ 为原 点, 所以也称 $\mathrm{CM}$ 框架. 而 GIA模型是在 $\mathrm{CE}$ 框架下建立 的，该框架以固体地球质心 $(\mathrm{CE})$ 为原点. 从理论上来 讲, 由于原点的不同, 两者存在一定的差异. Thomas等 (2011)分别估计了在ITRF2005的CM框架与ICE-5G的 CE框架下的 GPS测站垂向速度，发现在垂向速度的 $X 、 Y 、 Z$ 三个分量上分别只有 $(-0.2 \pm 0.1) 、(0.0 \pm 0.1)$ 和 $(-0.1 \pm 0.1) \mathrm{mm} \mathrm{a}^{-1}$ 的微小差别. Argus等(2014)认为GIA 引起的 $\mathrm{CM}$ 相对于 $\mathrm{CE}$ 的速度十分微小, 而现今冰质量 损失引起的 $\mathrm{CM}$ 相对于 $\mathrm{CE}$ 的速度相对比较明显, 如果 格陵兰岛冰量损失为 $200 \mathrm{Gt} \mathrm{a}^{-1}$ 且其他区域没有冰损 失, 该速度为 $0.22 \mathrm{~mm} \mathrm{a}^{-1}$. 这些改正量相对于GIA模型 较大的不确定性和GPS测站垂向速度的误差都十分微 小, 对本研究的影响可以忽略.

\section{3 地壳垂向运动因素的划分区域分析}

考虑到引起南极垂向运动的因素不完全是GIA, 还包括现今冰雪负荷变化和地质构造等因素. 目前针 对现今冰雪负荷变化引起的弹性响应的处理办法一般 有两种: 一种是通过建立弹性响应模型对GPS速度进 行弹性改正来消除现今冰雪负荷变化的影响, 另一种 方法是直接排除存在明显冰雪负荷变化的区域．鉴于 目前的弹性响应模型与GIA模型类似，还存在较大的 不确定性, 特别是在冰雪负荷变化显著的区域, 一般
在利用大地测量数据检验或约束GIA模型时都会排除 这些区域(Thomas等，2011；Ivins等，2013; Argus等， 2014), 而在冰雪负荷变化不显著的其他区域, 弹性响 应模型改正的影响又十分微小, 可以忽略不计(Argus 等, 2014). 因此, 本文决定采用剔除存在显著现今冰 雪负荷变化和活跃地质构造运动区域的测站数据, 以 消除或减弱它们的影响. 为了便于比较和分析不同因 素的影响, 本文根据各因素的影响程度和地理位置的 不同将南极大陆划分为若干区域, 主要包括南极半岛 北部、龙尼-菲尔希纳冰架、阿蒙森海沿岸、罗斯冰 架、埃里伯斯火山区域、西南极内陆和东南极沿海等 区域，根据有无明显的现今冰质量损失或者强烈的构 造活动来对每个区域的垂向运动特征进行讨论和分 析. 图3给出了本文58个南极GPS测站垂向速度与Argus等(2014)、Thomas等(2011)估计的GPS结果以及9 种GIA 模型预测结果在南极各区域的比较. 本文所用 到的GIA模型包括ICE-6G_C(VM5a)(Argus等，2014; Peltier等, 2015)、ICE-5G(VM2_L90)(Peltier, 2004; Argus和Peltier, 2010)、W12a(Whitehouse等, 2012a, 2012b)、Geruo13(Geruo等, 2013)、IJ05_R2(Ivins等, 2013)和Paulson07(Paulson等, 2007)等, 其中 Geruo13和 IJ05_R2模型由于地球参数不同存在多个版本. Greruo13的三个模型中(a)代表截断至100阶、无高斯平滑 的版本，(b)代表截断至60阶、200km高斯平滑的版本, (c)代表截断至40阶、500km高斯平滑的版本. IJ05_R2 $(65 \mathrm{~km})$ 代表岩石圈厚度为 $65 \mathrm{~km}$ 、下地幔黏滞度 $1.5 \times 10^{21} \mathrm{~Pa}$ s的版本, IJ05_R2 $(115 \mathrm{~km})$ 代表岩石圈厚度 


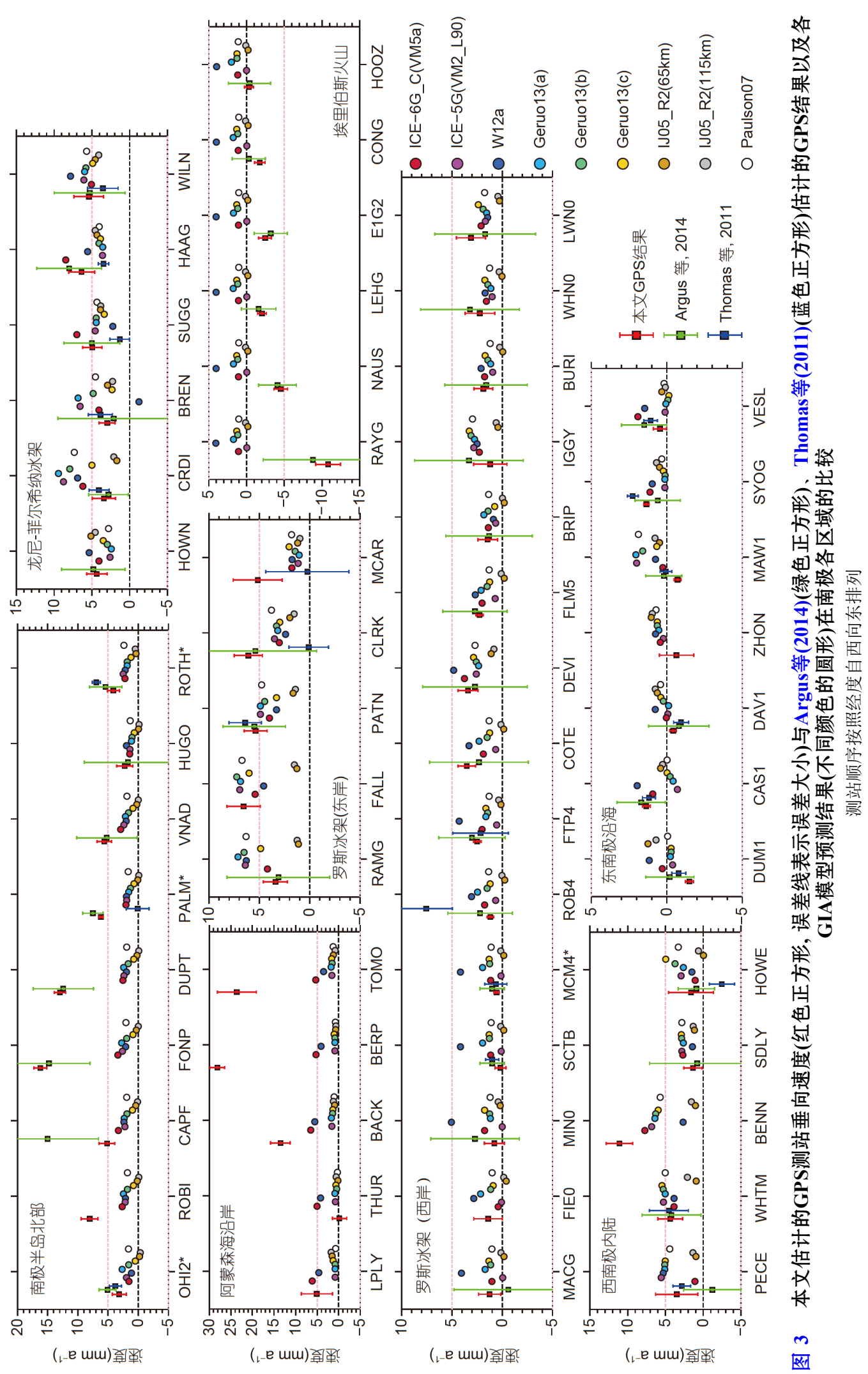


为 $115 \mathrm{~km}$ 、下地幔黏滞度 $4 \times 10^{21} \mathrm{~Pa} \mathrm{~s}$ 的版本. 本文与 $\mathrm{Ar}-$ gus等(2014)相同或并址的测站有47个, 与Thomas等 (2011)相同或并址的测站有 24 个, 即本文比他们多了 至少11个新测站．Argus等(2014)和Thomas等(2011)分 别采用了1994 2012年和1995 2010年的GPS数据, 而 本文采用了1996 2014年的GPS数据, 即相对较新的 GPS数据.

从图3中误差棒的长度可以看出, 本文GPS测站垂 向速度相比其他GPS结果精度有很大提高, 而且本文 结果与另外两种GPS结果具有较好的一致性, 但也有 个别测站结果存在分歧，如CAPF、ROB4等。测站 CAPF位于南极半岛北部，Argus等(2014)使用 2 年的数

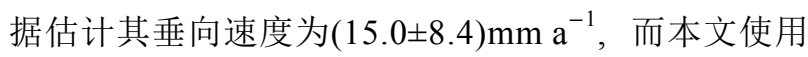
2010 2014年共计 5年的数据估计其速度为 $(5.18 \pm 1.29)$ $\mathrm{mm} \mathrm{a}^{-1}$, 两者相差达 $10 \mathrm{~mm} \mathrm{a}^{-1}$. 测站ROB4位于罗斯冰 架西岸, 本文估计其垂向速度为 $(1.17 \pm 0.33) \mathrm{mm} \mathrm{a}^{-1}$, 与 Argus等(2014)估计的( $2.22 \pm 3.20) \mathrm{mm} \mathrm{a}^{-1}$ 较为接近, 而 与Thomas等(2011)估计的(7.54 \pm 2.59$) \mathrm{mm} \mathrm{a}^{-1}$ 相差较大, 其中本文使用了 10年的数据, Argus等(2014)使用了6 年的数据, 而Thomas等(2011)仅使用了 558 天的数据. 上述差异说明测站数据时间跨度的长短对测站速度的 估计有十分重要的影响，过短的数据时间跨度会使速 度估计产生较大的不确定性.

\section{1 南极半岛北部}

南极半岛北部GPS测站垂向速度普遍大于各GIA 模型的预测值, 其中FONP测站垂向速度最大, 达到了 $16.19 \mathrm{~mm} \mathrm{a}^{-1}$, 而该测站的 GIA 模型预测值最大仅为 $3.37 \mathrm{~mm} \mathrm{a}^{-1}$, 这样大的差距不太可能由GIA模型不确 定性引起，因此该区域还存在其他因素引起的抬升运 动. 1995年南极半岛高兹塔王子道(Prince Gustav Channel)、拉森A冰架崩解, 2002年拉森B冰架崩解. 研究表明, 由于拉森B冰架的崩解, 原本处于冰架的包 围和阻挡中的冰河开始加速流失，该冰架的主要流入 源发生了重大质量亏损. 图4给出了南极半岛区域GPS 测站垂向速度分布, 测站FONP距离拉森B冰架崩解区 最近，且抬升速度最大，其他较远的测站速度相对较 小, 说明冰架崩解对该区域地壳抬升有很大影响. Thomas等(2011)通过对南极半岛北部OHI2、PALM和 ROTH等站点的坐标时间序列进行分析，发现坐标时 间序列在2002年前后存在明显不同的斜率(速度), 特

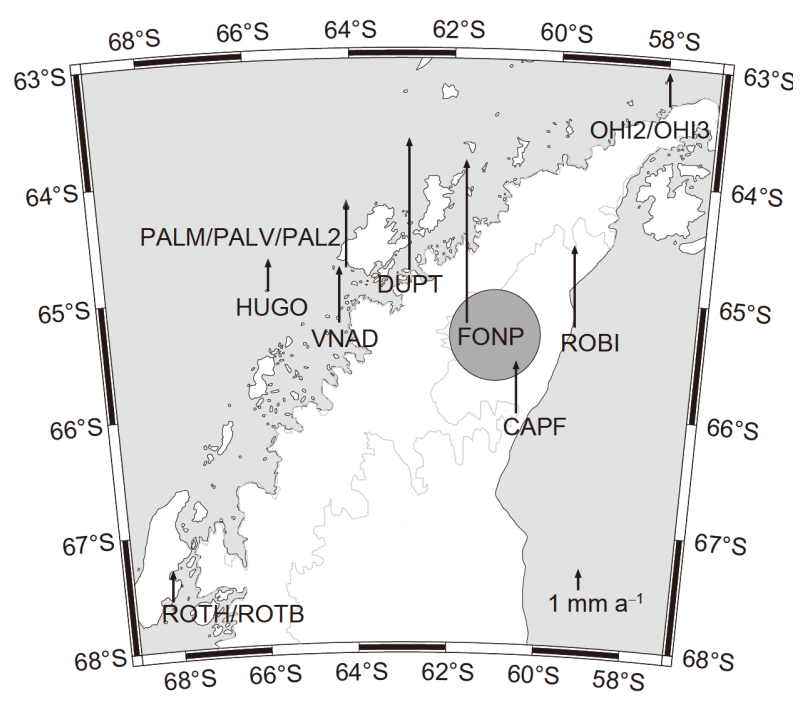

图 4 南极半岛地区GPS测站垂向速度分布

箭头, 方向代表抬升或下降, 大小代表速度量级, 灰线表示南极半岛 陆地边界线(不包括冰架), 灰色圆形区域表示2002年初崩解的拉森 B冰架近似范围

别是PALM站点2002年以前抬升速度仅为 $0.1 \mathrm{~mm} \mathrm{a}^{-1}$, 2002 年以后达到了 $8.8 \mathrm{~mm} \mathrm{a}^{-1}$. 本文估计的测站PALM 抬升速度变化如图5所示, 在1998 2001年期间抬升速 度为 $1.80 \mathrm{~mm} \mathrm{a}^{-1}$, 在2002 2014年期间抬升速度为 $6.08 \mathrm{~mm} \mathrm{a}^{-1}$, 比Thomas等(2011)计算的速度量级略小, 但速度在变大的趋势是一致的. 测站PALM在 2002 年 以前的垂向速度 $\left(1.8 \mathrm{~mm} \mathrm{a}^{-1}\right)$ 与各GIA模型预测速度吻 合较好, 说明 2002 年以前该测站垂向速度主要受GIA 的影响, 而受现今冰雪负荷变化的影响较小.

\section{2 龙尼-菲尔希纳冰架}

龙尼-菲尔希纳冰架区域的测站包括南极半岛南 部的1个测站(BREN)和龙尼-菲尔希纳冰架南部沿 岸 的 5 个测站. 在该区域本文的结果与Argus等(2014) 的结果十分相近, 而与Thomas等(2011)的结果在 SUGG、HAAG等测站上有较大差别, 原因可能与所 使用的数据时间跨度不同有关, Thomas等(2011)采用 了测站SUGG和HAAG 2003 2006年的GPS数据, 而本 文和Argus等(2014)都采用2006年之后的GPS数据. 该 区域GIA垂向速度预测值基本都在GPS测站垂向速度 处上下浮动, 本文的GPS结果与大多数GIA模型预测 值整体吻合较好, 因此本文认为龙尼-菲尔希纳冰架沿 岸区域地壳抬升的主要原因是GIA, 而不是现今冰雪 


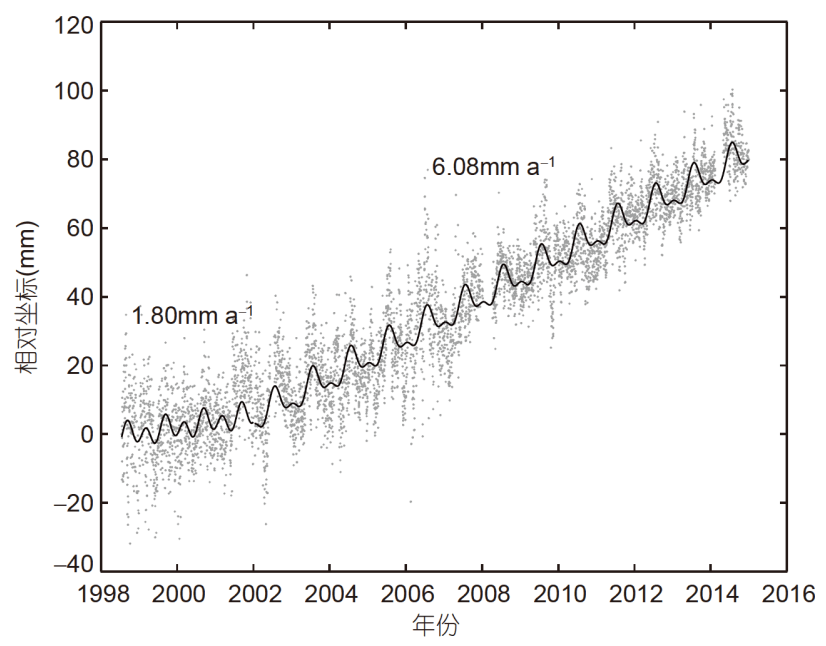

图 5 测站PALM在2002年前、后的垂向速度变化

负荷变化, 与Argus等(2014)得到的结论一致. 部分GIA 模型的预测速度在该区域存在较大分歧，在BREN测 站位置处可达约 $8 \mathrm{~mm} \mathrm{a}^{-1}$. 在CRDI、BERN等测站上 W12a、ICE-5G(VM2_L90)和Geruo13(a)模型预测值 与GPS结果相差较大, 说明这些GIA模型在该区域存 在较大的不确定性.

\section{3 阿蒙森海沿岸}

由于本文在阿蒙森海沿岸区域的 5 个测站均为 2011年之后建立, 所以Argus等(2014)和Thomas等 (2011)的分析中均未引入这些测站，本文采用这些测 站的数据时间跨度为 $3 \sim 4$ 年, 能够满足地壳运动速度 估计的要求. 在阿蒙森海沿岸区域GPS测站垂向速度 范围为 $-0.17 \mathrm{~mm} \mathrm{a}^{-1}$ (THUR) 28.13 $\mathrm{mm} \mathrm{a}^{-1}$ (BERP), 不 同GPS测站垂向速度之间存在较大差异，而各GIA模 型预测值在该区域较为稳定，其中ICE-6G_C(VM5a) 和W12a模型预测相对偏大, 在 $5 \mathrm{~mm} \mathrm{a}^{-1}$ 左右, 其他GIA 模型预测值相对较小, 在 $0 \sim 2 \mathrm{~mm} \mathrm{a}^{-1}$. LPLY和THUR测 站与各GIA模型整体吻合较好, 说明其垂向速度主要 与GIA有关; BACK、BERP和TOMO三个测站的抬升 速度远远大于所有GIA模型预测值，可能与该区域明 显的现今冰雪负荷变化有关.

测站BACK、BERP和TOMO均处于派恩艾兰湾 区域，研究表明该区域的两大冰川派恩艾兰冰川和思 韦茨冰川都在经历快速的冰雪质量损失. 派恩艾兰冰 川是南极众多冰川中最大、移动速度最快的冰川,
2004年卫星探测结果显示表面的冰块流入阿蒙森海沿 岸(Amundsen Sea)的速度比 30 年前要快 $25 \%$. 思韦茨冰 川底部隐藏着庞大的快速流动低洼水道网络, 是西南 极最大的冰流之一. 该区域是整个南极冰质量损失最 大的区域，这三个测站异常大的速度较好地反映了该 区域现今冰质量损失引起的弹性抬升.

\section{4 埃里伯斯火山}

埃里伯斯火山位于罗斯冰架西南部的罗斯岛, 是 一座活火山. 火山口底部是一个小熔岩湖，1984年9月 17日喷发, 将火山熔岩抛出主火山口, 该区域地震台站 曾纪录到许多小型的地震, 至今该火山仍然是研究强 地质现象的重要对象. 研究发现, 地震有可能导致火 山附近地表下沉. Takada和Fukushima(2013)和Pritch$\operatorname{ard}(2010)$ 等利用卫星数据分别分析了由 2011 年日本东 北 9.0 级地震和 2010 年智利莫莱 8.8 级地震所造成的地 表形变, 在两次地震后, 在断层附近的火山下沉程度最 高达到 $15 \mathrm{~cm}$, 其中日本火山的下沉很可能是由于火山 下面的岩浆储存区以及一些较脆热岩的下沉所导致, 而智利火山的下沉则可能是因为火山下面热流体的释 放所导致. 由于埃里伯斯火山附近的测站可能受到地 下岩浆作用的影响, 本文将火山附近 $3 \mathrm{~km}$ 内的 RAYG、NAUS、LEGH、E1G2、CONG和HOOZ 6 个 测站从罗斯冰架区域提取出来单独进行分析. 由图3可 知, 该区域GPS测站均在下沉, 其中位于主火山口的测 站RAYG下沉最为严重, 速度达 $(-10.80 \pm 1.62) \mathrm{mm} \mathrm{a}^{-1}$, 下沉速度随着到火山口的距离增大而减小, 测站 HOOZ距火山口约 $3 \mathrm{~km}$, 其垂向速度为 $(-0.32 \pm 0.57)$ $\mathrm{mm} \mathrm{a}^{-1}$, 在距离火山口约 $28 \mathrm{~km}$ 的测站 $\mathrm{MCM} 4 *$ 变为抬 升运动, 速度为 $(0.24 \pm 0.26) \mathrm{mm} \mathrm{a}^{-1}$. 该区域各GIA模型 预测结果均为抬升运动, W12a模型预测值量级偏大, 平均在 $4 \mathrm{~mm} \mathrm{a}^{-1}$ 左右, 其他模型预测值在 $0 \sim 2 \mathrm{~mm} \mathrm{a}^{-1}$. 该区域GPS实测垂向速度(负值)与GIA预测速度(正值) 相差较大, 再加上不存在较大的现今冰雪负荷变化, 说 明该区域的下沉并非GIA和现今冰雪负荷变化引起, 而是火山底部岩浆活动的结果.

\section{5 罗斯冰架}

除去火山附近的 6 个测站, 罗斯冰架西岸其余GPS 测站均为抬升, 最大速度为 $3.53 \mathrm{~mm} \mathrm{a}^{-1}$ (COTE), 与大部 分GIA模型预测值总体吻合较好, 说明该区域的抬升 
运动主要受到GIA的影响. 罗斯冰架东岸的GPS测站 抬升较为平稳且速度平均比西岸稍大, 在 $5 \mathrm{~mm} \mathrm{a}^{-1}$ 左 右, 测站RAMG、FALL和PATN垂向速度与GIA模型 预测值吻合较好, 可认为这三个测站所在位置的抬升 主要由GIA引起. 对于测站CLRK和MCAR, 本文分别 使用 5 年和 7 年的GPS数据估计垂向速度和误差分别为 (6.11 \pm 1.40$)$ 和(5.16 \pm 2.44$) \mathrm{mm} \mathrm{a}^{-1}$, 分别比GIA模型最大 预测值大了 2.32 和 $3.35 \mathrm{~mm} \mathrm{a}^{-1}$, 也超出了 GPS测站垂向 速度估计的误差范围, 因此不应该简单认为是GPS速 度估计的误差. Thomas等(2011)估计的这两个测站的 速度接近于 $0 \mathrm{~mm} \mathrm{a}^{-1}$, 与本文和Argus等(2014)的结果 相差较大, 原因可能是由于估计所使用的数据时间跨 度不同, 前者采用1998 2002年不到200天的GPS数据, 而后两者均采用 2010 年之后的连续GPS测站数据. 测 站 CLRK和MCAR分别位于玛丽伯德地西北部的 Clarke山脉和Carbone山, 该地区地质构造稳定且尚未 发现明显的现今冰雪负荷变化, 因此有可能是GIA模 型系统性地低估了该地区的GIA抬升. 两种IJ05_R2模 型对罗斯冰架西岸的地壳垂向速度预测值普遍低于本 文GPS测站垂向速度和其他GIA模型, 说明它们在该 区域可能存在较大的不确定性.

\section{6 西南极内陆}

测站PECE、WHTM、SDLY和HOWE位于西南 极内陆, GPS抬升速度都小于 $5 \mathrm{~mm} \mathrm{a}^{-1}$, 与大多数GIA 模型吻合较好. 在BENN测站位置处各GIA模型差别 较大, 最大差别可达 $7 \mathrm{~mm} \mathrm{a}^{-1}$, 表明在位置上不同GIA 模型预测之间存在较大的不确定性. 该测站位于班尼 特冰原岛峰, 于 2010 年 12 月建立, 数据时间跨度较短, 此前有关GIA建模的研究中都未曾使用该测站的数据 来约束和检验GIA模型, 本文利用2010 2014年的GPS 数据估计其垂向速度为 $(11.09 \pm 1.75) \mathrm{mm} \mathrm{a}^{-1}$, 比所有 GIA模型预测速度都大得多, 与GIA模型预测值的最 小不符值为 $3.35 \mathrm{~mm} \mathrm{a}^{-1}$ (ICE6G_C(VM5a)), 最大不符 值为 $10.07 \mathrm{~mm} \mathrm{a}^{-1}$ (IJ05_R2 $\left.(65 \mathrm{~km})\right)$. 图6给出了该测站 的坐标时间序列, 可以看出其时间序列呈现平稳上升 趋势, 观测期间无明显阶跃, 且红色曲线对线性项和 周期项的拟合效果较好, 因此该测站的速度可靠性较 高. 该测站所在地区尚未发现明显的冰质量损失, 因 此可以不考虑现今冰雪负荷变化的影响, GPS抬升速 度明显大于GIA模型预测值的原因有可能是各GIA模
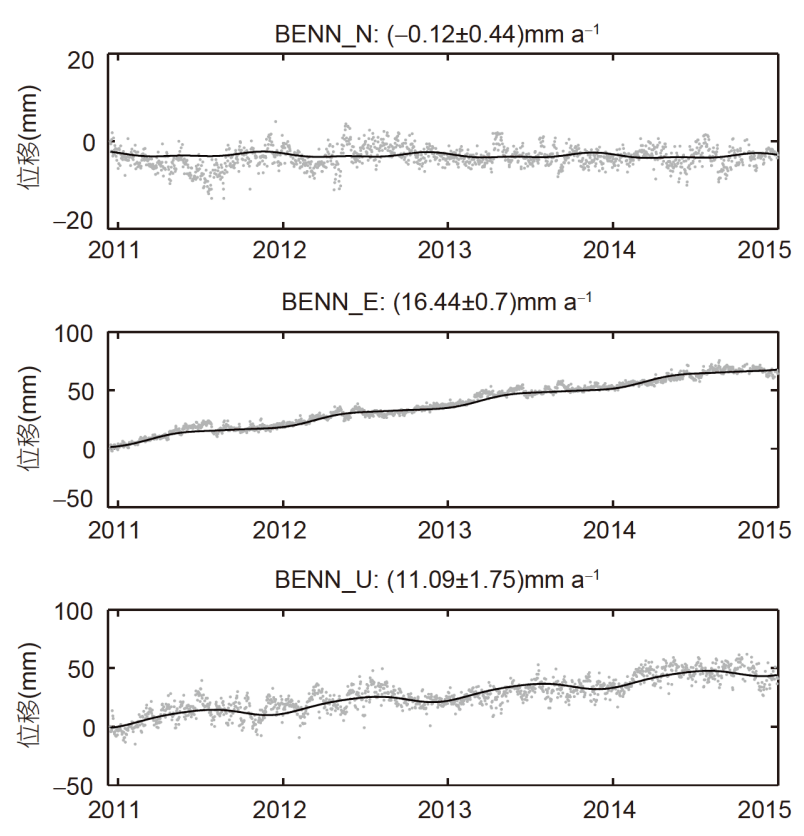

图 6 测站BENN的坐标时间序列以及速度估计 黑线代表对线性项和周期项的拟合结果

型低估了该位置的GIA抬升量级.

\section{7 东南极沿海}

东南极沿海区域 GPS 测站垂向速度在-1.5 $1.35 \mathrm{~mm} \mathrm{a}^{-1}$, 平均量级比西南极区域测站偏小, 该区域 各GIA模型预测速度量级也相对较小, 约为 $-1 \sim 3 \mathrm{~mm}$ $\mathrm{a}^{-1}$. 东南极基底是古老的克拉通, 地质构造十分稳定, 所以该区域基本不存在地质构造因素引起的垂向运 动. GRACE重力数据表明从2009年1月到2013年2月毛 德皇后地东部和恩德比地的冰雪积累速度约为 $150 \mathrm{Gt}$ $\mathrm{a}^{-1}$ (Boening等, 2012; Argus等, 2014), 总计约600Gt. 沉 积数据也表明2009 2012年该区域冰雪快速积累, 但测 得1980 2008年总积累量约为0Gt, 说明最近的冰雪积 累速度相比2008年以前是十分反常的. 2009 2012年 冰雪负荷积累会引起海岸下沉, 各GIA模型预测普遍 为轻微的抬升运动, 位于恩德比地东部沿海的MAW1 测站垂向速度为 $(-0.72 \pm 0.23) \mathrm{mm} \mathrm{a}^{-1}$, 能够反映该区域 冰雪积累引起的轻微下沉现象, 但无法排除各GIA模 型可能存在较大的不确定性. 位于拉斯曼丘陵的 ZHON和DAV1站以及位于迪蒙迪维尔海边的DUM1 站也存在轻微下沉, 速度略小于各GIA模型的预测值, 具体原因还有待于进一步调查. 位于毛德皇后地北部 
的VESL和SYOG站以及位于温森斯湾的 CAS 1 站的 GPS速度均为正值, VESL和SYOG站速度与各GIA模 型预测值吻合较好, CAS1站速度与ICE-6G(VM5a)和 $\mathrm{W} 12 \mathrm{a}$ 模型吻合较好, 与其他模型的一致性较差. 总体 来说, 东南极沿海区域测站垂向运动并不显著, 受GIA 和现今冰雪负荷变化的影响都比较小.

\section{GIA模型预测的检核与分析}

为了直观地看出GPS测站垂向速度与各GIA模型 的差异, 在图7中给出了南极大陆58个GPS测站垂向速 度与9种GIA 模型预测之间的比较, 其中各种颜色的圆 圈代表GPS速度, 背景色代表GIA模型, 不同的颜色代 表不同的速度量级，颜色越接近说明GPS速度与GIA 模型吻合程度越高. 由图7可以看出在南极半岛、阿 蒙森海沿岸派恩艾兰湾和埃尔伯斯火山区域的测站颜 色与各GIA模型背景颜色都相差很大, 而在其他区域 (特别是东南极)测站颜色与背景色相差较小, 原因在 于南极半岛、阿蒙森海沿岸派恩艾兰湾和埃尔伯斯火 山区域地壳除了受到GIA作用, 还受到明显的现今冰 雪负荷变化或地下岩浆活动的影响. 为了消除或减弱 这两种因素的影响, 本文在利用GPS测站垂向速度检 核各GIA模型预测值时, 排除了南极半岛北部9个 测 站、派恩艾兰湾区域 3 个测站 (BACK、BERP和 $\mathrm{TOMO}$ )以及埃里伯斯火山附近 6 个测站, 剩余的 40 个 测站都处于现今冰雪负荷变化并不显著并且地质构造 相对稳定的区域.

ICE-5G(VM2_L90)、Geruo13(a/b/c) 和Paulson07 模型预测速度的空间分布十分相似, 这是因为它们都 采用了相同的冰模型ICE-5G, 与采用其他冰模型的 GIA模型(如ICE-6G(VM5a)、IJ05_R2和W12a)相比在 分布和量级上都存在较大差异, 说明冰模型在GIA模 型的预测结果中起主导作用. ICE-5G(VM2_L90)、 Geruo13(a/b/c) 和Paulson07模型预测结果的微小差异 主要来自不同的地球模型, ICE-5G(VM2_L90)模型使 用了VM2 黏滞度剖面和岩石圈厚度为 $90 \mathrm{~km}$ 的地球模 型, Paulson07模型使用的近似VM2的四层黏滞度模型, Geruo13(a/b/c)采用VM2黏滞度剖面和三维可压缩地 球模型. 值得一提的是, 这些地球模型都是黏滞度仅沿 径向变化的一维模型, 并没有考虑黏滞度的横向不均 匀性. 为了探讨黏滞度横向非均匀性对GIA预测的影
响，汪汉胜等(2009)通过建立大地测量观测和相对海 平面联合约束的GIA模型ICE-4G+RF3L20 $(\beta=0.4)$, 发 现地幔黏滞度具有显著的横向非均匀性并且对GIA预 测有显著影响, 这种横向不均匀的黏滞度可以由地震 层析模型得到. Wilson等(2015)的地震结果也表明南 极地幔黏滞度具有显著且复杂的横向变化, 这种横向 变化从根本上影响到对南极冰雪质量变化的均衡响 应. 鉴于目前包括ICE-6G(VM5a)在内的大多数GIA模 型都没有考虑黏滞度的横向不均匀性, 在今后的GIA 模型研究中, 确定真实的三维地幔黏滞度结构对于 GIA理论发展和模型改进具有重要的意义.

\subsection{GPS测站垂向速度与GIA模型预测的相关性分 析}

为了评价GPS测站垂向速度和各GIA模型预测值 的相关性, 本文对GPS测站垂向速度和各GIA模型预 测值线性拟合, 拟合效果如图8所示, 可以看出在所有 结果中都存在一个偏离拟合直线最远的测站BENN; 本文在3.6节也提到在该测站位置上GPS测站垂向速 度比GIA模型预测值偏大得多, 原因可能是大多数GIA 模型在该测站位置缺乏实测数据约束. 由表 2 可以看 出, GPS测站垂向速度与各GIA模型预测值拟合的直 线斜率在 $0.45 \sim 1.13$; 截距在 $0.26 \sim 1.41$; 相关系数在 0.35 0.76. 从理论上来讲如果GPS测站垂向速度仅包 含GIA的响应, 那么图8、表2中的斜率和截距都分别 为 1 和 0 , 而相关系数都为 1 . 反过来讲, 斜率越接近 1 、 截距越接近 0 、相关系数越大则说明GPS测站垂向速 度与该GIA模型预测值的吻合程度越高, GPS测站垂 向速度与ICE-6G_C(VM5a)拟合的截距 $\left(0.26 \mathrm{~mm} \mathrm{a}^{-1}\right)$ 最 小且相关系数 (0.76)最大, 与Geruo13(c) 拟合的斜率最 接近于 $1(1.01)$ 且相关系数 $(0.72)$ 仅次于 ICE-6G_C (VM5a), 说明GPS实测速度与这两个GIA模型预测值 吻合性最好. GPS测站垂向速度与GIA模型预测的线 性拟合和相关系数在一定程度上能够反映GIA模型的 不确定性, 接下来我们对各GIA模型的不确定性集中 进行分析.

\subsection{GIA模型不确定性分析}

GIA模型的不确定性主要来自两个方面: 一是冰 模型中冰盖厚度历史不够精确, 二是地球模型中有相 当大的简化. 同为古冰盖区域的劳伦古大陆或芬诺斯 


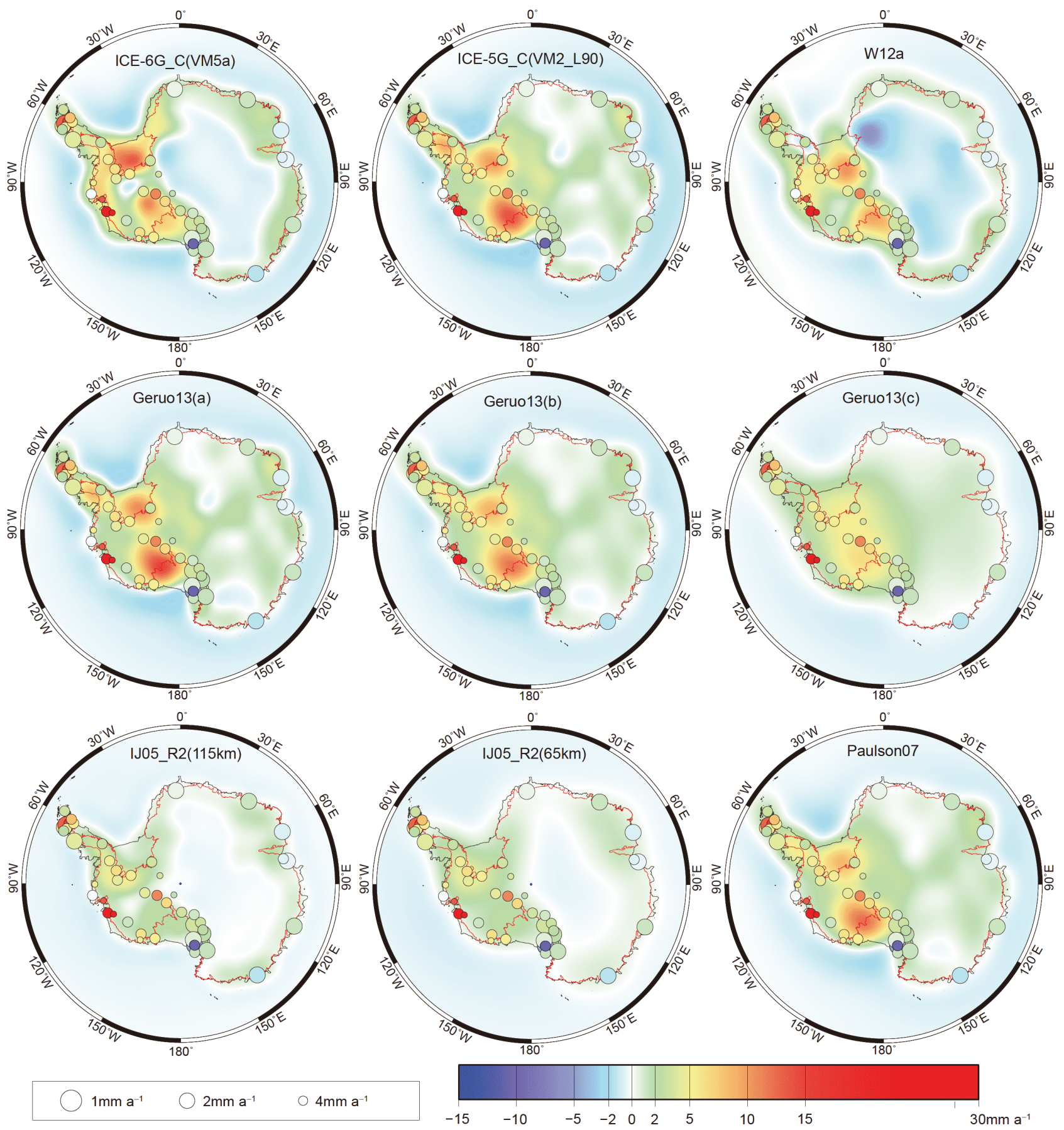

图 7 本文GPS测站垂向速度(圆圈)与各GIA模型(背景)比较

不同颜色表示GPS速度或模型预测速度的大小, 圆圈的大小表示实测速度精度

坎底亚地区现今都是内海，分别为哈德逊湾和波的尼 亚湾，其陆地在冰期后出现了许多全新世的海岸线记 录，对冰消历史和地幔黏滞度具有强烈的约束. 相比 之下，南极地区缺乏过去冰盖最大厚度区域的精确
RSL历史资料, 会导致冰模型构建存在非唯一性, 并且 南极地区用于约束模型的大地测量数据较少且存在现 今冰雪负荷变化引起的弹性响应，南极区域冰量损失 历史的重建问题比劳伦古大陆或芬诺斯坎底亚地区都 

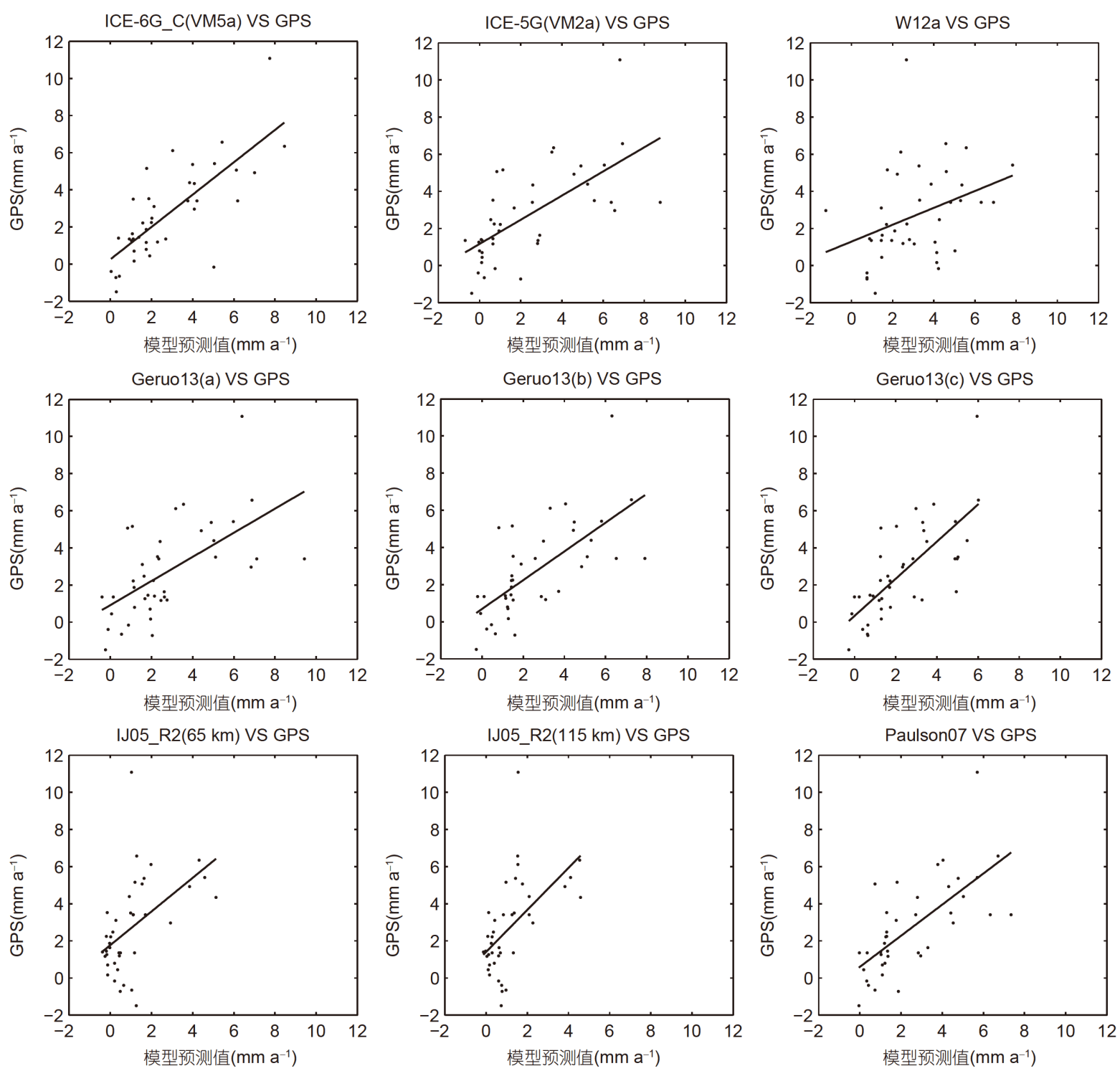

图 8 GPS测站垂向速度与各GIA模型预测的线性拟合效果

更加复杂. 一直以来, GIA模型的误差要么没有给出, 要么使用模型之间的差别来量化误差, 如W12a模型量 化误差时使用了一系列不同的冰模型版本, Geruo13、 IJ05_R2模型在量化误差时也使用了一系列不同参数 的地球模型，但无论哪一种方法都无法准确地反映真 实的不确定性，特别是当对GRACE数据进行GIA改正 时, 将导致系统误差, 而不是随机误差.

为了分析不同GIA模型的真实不确定性，我们以 GPS测站垂向速度作为参考，求出GPS测站垂向速度 与各GIA模型之间的不符值，并统计不符值在不同区
间的个数. 考虑到东南极和西南极之间的较大差异, 图9分别给出了东南极和西南极GPS测站垂向速度与 各GIA模型预测速度的不符值分布，各区间长度均为 $1 \mathrm{~mm} \mathrm{a}^{-1}$. 东南极测站包括东南极沿海 7 个测站和罗斯 冰架西岸的 15 个测站，共计 22 个测站. 西南极测站包 括龙尼-菲尔希纳冰架 6 个测站、阿蒙森海沿岸 2 个测 站(除去派恩艾兰湾)、罗斯冰架东岸 5 个测站和西南 极内陆 5 个测站, 共计 18 个测站. 由图 9 可知, 东南极的 不符值分布较为集中，不符值范围约在-4 4 $\mathrm{mm} \mathrm{a}^{-1}$, 而西南极不符值分布较为分散，不符值范围约在 
表 2 GPS测站垂向速度与各GIA模型预测的线性拟合参 数和相关系数

\begin{tabular}{cccc}
\hline GIA模型名称 & 拟合斜率 & $\begin{array}{c}\text { 拟合截距 } \\
\left(\mathrm{mm} \mathrm{a}^{-1}\right)\end{array}$ & 相关系数 \\
\hline ICE-6G_C(VM5a) & 0.87 & 0.26 & 0.76 \\
ICE-5G(VM2_L90) & 0.65 & 1.17 & 0.66 \\
W12a & 0.45 & 1.29 & 0.35 \\
Geruo13(a) & 0.65 & 0.92 & 0.60 \\
Geruo13(b) & 0.77 & 0.69 & 0.68 \\
Geruo13(c) & 1.01 & 0.33 & 0.72 \\
IJ05_R2(65km) & 0.91 & 1.77 & 0.50 \\
IJ05_R2(115km) & 1.13 & 1.41 & 0.56 \\
Paulson07 & 0.84 & 0.60 & 0.69 \\
\hline
\end{tabular}

$-6 \sim 10 \mathrm{~mm} \mathrm{a}^{-1}$, 说明各GIA模型在西南极的不确定性普 遍要大于东南极.

表3给出了GPS测站垂向速度与各GIA模型预测 速度不符值的加权均值和加权均方根误差(WRMS)的 比较，不符值的加权均值可以检验两者是否有无系统 性偏差，不符值的WRMS能够有效衡量两者之间偏差 量级的大小. 由表 3 可以发现, 不符值的加权均值在西 南极均 $\geq 0$, 说明在西南极GPS结果比各GIA 模型预测 都偏大，而在东南极既有正值也有负值，负值表明在 东南极ICE-6G_C(VM5a)、W12a、Geruo13(a)模型预 测比GPS结果偏大. GPS垂向速度与IJ05_R2 $(65 \mathrm{~km})$ 、 IJ05_R2 $(115 \mathrm{~km})$ 模型不符值的总体加权均值范围为 1.55 和 $1.67 \mathrm{~mm} \mathrm{a}^{-1}$, 说明这两个GIA模型预测比GPS结 果系统性地偏小, 与W12a模型不符值的总体加权均值
为 $-0.39 \mathrm{~mm} \mathrm{a}^{-1}$, 说明W12a 模型预测比GPS结果系统 性地偏大，与ICE-6G_C(VM5a)、ICE-5G(VM2 L90)、Geruo13系列和Paulson07模型预测速度不符值 的加权均值量级相对较小, 说明这些模型预测速度与 GPS垂向速度的总体无偏性较好. 西南极GPS测站垂 向速度与GIA模型预测不符值的WRMS普遍大于东南 极, 与图9中得出的结论一致. GPS测站垂向速度与 ICE-6G_C(VM5a)不符值的WRMS $\left(0.88 \mathrm{~mm} \mathrm{a}^{-1}\right)$ 最小, 其次是与Geruo13(c)不符值的WRMS $\left(1.15 \mathrm{~mm} \mathrm{a}^{-1}\right)$, 与 IJ 05_R2 两个模型预测不符值的 WRMS ( 2.10 和 $\left.2.01 \mathrm{~mm} \mathrm{a}^{-1}\right)$ 最大, 因此以 GPS垂向速度作为参考, 本文 认为在众多GIA模型中ICE-6G_C (VM5a)模型在上述 区域的不确定性最小, 其次是Geruo13(c)模型, IJ05_R2 两个模型的不确定性最大. ICE-6G_C (VM5a)模型不确 定性最小的原因可能与约束模型所使用的数据集有 关，该模型采用了Argus等(2014)估计的42个GPS测站 垂向速度进行约束，而其他模型如W12a、IJ05_R2等 则采用较早的Thomas等(2011)GPS数据集来进行约束.

不同地球模型参数设置可能会对GIA模型的不确 定性造成一定影响. Geruo13系列三个模型主要是截 断阶数和高斯滤波半径不同, 从而导致三种不同的预 测结果. 由表 3 中不符值的WRMS和表 2 中的相关系数 可以看出随着截断阶次越低、高斯滤波半径越大, WRMS越小, 相关系数越高, 即预测速度越接近GPS 测站垂向速度, 原因可能是模型高阶项含有更大的误 差, 通过截断到较低的阶次和高斯滤波能够有效减小 这种误差. IJ05_R2系列两个模型主要是岩石圈厚度 和下地幔黏滞度的不同, 由表3中不符值的WRMS和

表 3 GPS测站垂向速度与模型预测不符值的加权均值和加权均方根误差(WRMS)比较

\begin{tabular}{|c|c|c|c|c|c|c|}
\hline \multirow{2}{*}{ GPS-GIA } & \multicolumn{3}{|c|}{ 不符值加权均值 $\left(\mathrm{mm} \mathrm{a}^{-1}\right)$} & \multicolumn{3}{|c|}{ 不符值WRMS $\left(\mathrm{mm} \mathrm{a}^{-1}\right)$} \\
\hline & 东南极 & 西南极 & 总体 & 东南极 & 西南极 & 总体 \\
\hline GPS-ICE-6G_C(VM5a) & -0.18 & 0.00 & -0.10 & 0.75 & 2.08 & 0.88 \\
\hline GPS-ICE-5G(VM2_L90) & 0.59 & 0.04 & 0.34 & 1.48 & 2.59 & 1.53 \\
\hline GPS-W12a & -1.27 & 0.68 & -0.39 & 1.26 & 3.08 & 1.67 \\
\hline GPS-Geruo13(a) & -0.15 & 0.09 & -0.04 & 1.42 & 2.85 & 1.53 \\
\hline GPS-Geruo13(b) & 0.04 & 0.17 & 0.10 & 1.26 & 2.32 & 1.33 \\
\hline GPS-Geruo13(c) & 0.03 & 0.69 & 0.33 & 1.05 & 1.89 & 1.15 \\
\hline GPS-IJ05_R2 $(65 \mathrm{~km})$ & 0.98 & 2.51 & 1.67 & 1.67 & 2.42 & 2.10 \\
\hline GPS-IJ05_R2(115km) & 0.85 & 2.41 & 1.55 & 1.55 & 2.28 & 2.01 \\
\hline GPS-Paulson07 & 0.06 & 0.35 & 0.19 & 1.31 & 2.22 & 1.39 \\
\hline
\end{tabular}



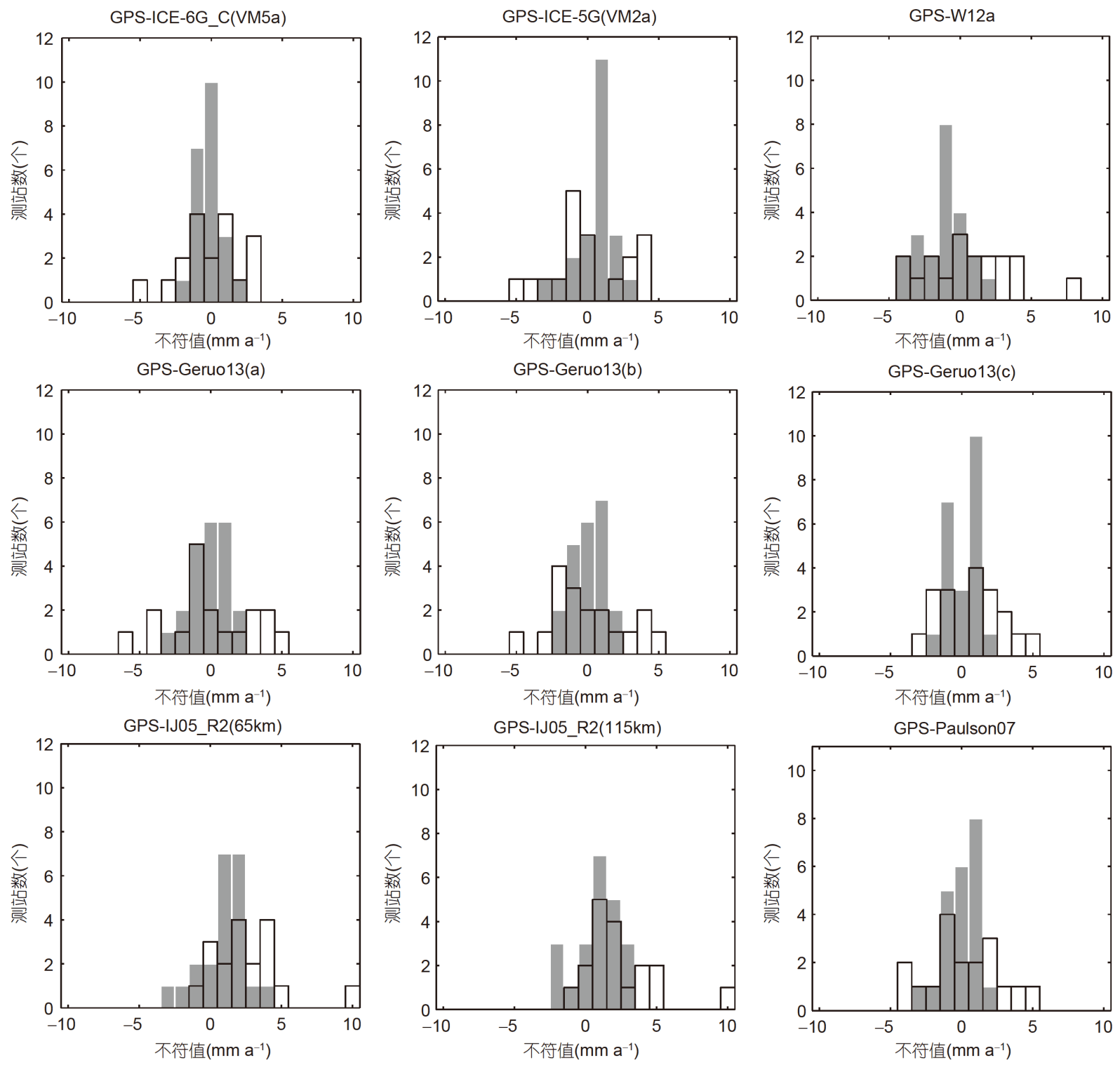

图 9 GPS测站垂向速度与模型预测在东南极(灰色柱体)和西南极(无色柱体)的不符值分布

表2中的相关系数可以看出, 岩石圈厚度较大、下地幔 黏滞度较大的IJ05_R2 (115km)模型预测结果更接近 GPS结果.

虽然目前南极GIA模型相比以前版本的模型有了 很大改进，如ICE-6G C (VM5a)相对于ICE-5G (VM2_L90)的不确定性有了显著减小，但是南极GIA 建模的状况仍然不容乐观．在北美地区，Park等(2002) 计算的GPS结果与改进地球模型的ICE-3G模型预测不 符值的WRMS为 $0.42 \mathrm{~mm} \mathrm{a}^{-1}$, 朱新慧和孙付平(2005)利 用VLBI结果与ICE-4G模型的相关系数为 0.89 , 不符值
WRMS 为 $0.8 \mathrm{~mm} \mathrm{a}^{-1}$, 目前最新的ICE-6G C (VM5a)模 型相比ICE-3G、ICE-4G模型都有了很大改进(Peltier 等, 2015). 而在南极地区GPS结果与各GIA模型的相关 系数范围在 $0.35 \sim 0.76$, 不符值 WRMS 范围在 0.88 2.10 $\mathrm{mm} \mathrm{a}^{-1}$. 因此，与北美地区相比，南极地区 GIA模型预测与实测垂向速度相关系数普遍不高, 并 且不符值WRMS偏大. 在现今冰雪负荷变化不显著地 区的部分测站上(如BENN等)与GPS垂向速度存在较 大不符值, 而在现今冰雪负荷变化显著地区(如南极半 岛北部和阿蒙森海沿岸派恩艾兰湾)缺乏直接GPS约 
束, 弹性改正模型不确定性偏大.

\section{5 结论}

利用GAMIT/GIOBK 10.5 软件解算了南极及周边 区域1996 2014年73个GPS测站的数据, 并联合全球网 IGS1 IGS9的基线解算数据( $\mathrm{h}$ 文件)进行联合平差，得 到各测站的单天坐标时间序列. 采用三倍四分位数差 法则剔除了坐标时间序列中的异常值，并对部分测站 时间序列中存在的阶跃使用CATS软件进行了估计并 改正，考虑白噪声、闪柏噪声和随机漫步噪声采用 CATS 软件中的最大似然方法估计了各测站速度及不 确定性, 对并址站或相近测站的速度进行了合并.

本文GPS测站垂向速度与Argus等(2014)、Thomas等(2011)估计的GPS测站垂向速度以及各GIA模型 预测结果的分区域分析结果表明，在南极半岛北部和 阿蒙森海沿岸派恩艾兰湾地区存在现今冰雪负荷变化 引起的显著弹性抬升, 在埃尔伯斯火山区域存在显著 的地壳下沉，可能与火山地下岩浆活动有关，东南极 沿海测站垂向运动速度都不显著，其他区域的测站垂 向运动主要受到GIA的影响.

通过对本文GPS结果与GIA模型的相关性以及模 型不确定性进行分析, 发现ICE-6G(VM5a)模型与本文 GPS测站垂向速度的一致性最好，其次是Geruo13模 型，而W12a和IJ05_R2系列模型与本文GPS结果一致 性相对较差. 目前新出现的南极GIA模型相比之前的 模型都有很大改善，但南极GIA建模的状况与北美等 其他地区相比仍然不容乐观，随着未来南极大地测量 数据集的不断扩充、地幔黏滞度横向非均匀性等GIA 理论地研究以及建模方法的改进，这一状况将会得到 改善.

\section{参考文献}

鄂栋臣, 张胜凯. 2006. 国际南极大地参考框架的构建与进展. 大地 测量与地球动力学, 26: 104-108

姜卫平, 周晓慧. 2014. 澳大利亚 GPS 坐标时间序列跨度对噪声模型 建立的影响分析. 中国科学: 地球科学, 44: 2461-2478

姜卫平, 赵倩, 刘鸿飞, 杨凯. 2011. 子网划分在大规模GNSS基准站 网数据处理中的应用. 武汉大学学报(信息科学版), 35: 389-391

李伟伟, 鞠晓蕾, 沈云中, 张子占. 2014. 联合GNSS和GRACE数据分 析南极跟踪站的径向形变. 极地研究, 26: 238-243
李斐, 马超, 张胜凯, 雷锦韬, 郝卫峰, 张卿川, 李文浩. 2016. 南极半 岛地区GPS坐标时间序列噪声分析及形变模式初探. 地球物理学 报, 59: 2402-2412

马超, 李斐, 张胜凯, 雷锦搯, 鄂栋臣, 郝卫峰, 张卿川. 2016. 顾及共 性误差的南极半岛地区连续GPS站坐标时间序列分析. 地球物理 学报, 59: 2783-2795

汪汉胜, Patrick W U, Wouter V D W, 贾路路, 胡波, 王志勇. 2009. 大 地测量观测和相对海平面联合约束的冰川均衡调整模型. 地球 物理学报, 52: 2450-2460

朱新慧, 孙付平. 2005 . 用甚长基线干涉测量数据检测冰期后地壳回 弹. 地球物理学报, 48: 308-313

Altamimi Z, Collilieux X. 2009. IGS contribution to the ITRF. J Geod, 83: $375-383$

Argus D F, Peltier W R, Drummond R, Moore A W. 2014. The Antarctica component of postglacial rebound model ICE-6G_C (VM5a) based on GPS positioning, exposure age dating of ice thicknesses, and relative sea level histories. Geophys J Int, 198: 537-563

Argus D F, Peltier W R. 2010. Constraining models of postglacial rebound using space geodesy: A detailed assessment of model ICE5G (VM2) and its relatives. Geophys J Int, 107: 697-723

Blewitt G, Lavallée D. 2003. Effect of annual signals on geodetic velocity. J Geophys Res, 108: 2010

Boening C, Lebsock M, Landerer F, Stephens G. 2012. Snowfall-driven mass change on the East Antarctic ice sheet. Geophys Res Lett, 39: L21501

Geruo A, Wahr J, Zhong S. 2013. Computations of the viscoelastic response of a 3-D compressible Earth to surface loading: An application to Glacial Isostatic Adjustment in Antarctica and Canada. Geophys J Int, 192: 557-572

Ivins E R, James T S, Wahr J, Schrama O E J, Landerer F W, Simon K M. 2013. Antarctic contribution to sea level rise observed by GRACE with improved GIA correction. J Geophys Res-Solid Earth, 118: 3126-3141

King M A, Altamimi Z, Boehm J, Bos M, Dach R, Elosegui P, Fund F, Hernández-Pajares M, Lavallee D, Mendes Cerveira P J, Penna N, Riva R E M, Steigenberger P, van Dam T, Vittuari L, Williams S, Willis P. 2010. Improved constraints on models of glacial isostatic adjustment: A review of the contribution of ground-based geodetic observations. Surv Geophys, 31: 465-507

King M A, Santamaría-Gómez A. 2016. Ongoing deformation of Antarctica following recent great earthquakes. Geophys Res Lett, 43: 1918-1927

Milne G A, Davis J L, Mitrovica J X, Scherneck H G, Johansson J M, Vermeer M, Koivula H. 2001. Space-geodetic constraints on glacial isostatic adjustment in Fennoscandia. Science, 291: 2381-2385 
Mitrovica J X, Gomez N, Clark P U. 2009. The sea-level fingerprint of West Antarctic collapse. Science, 323: 753

Nield G, Whitehouse P, King M, Clarke P. 2015. Glacial Isostatic Adjustment on the Siple Coast. Vienna: EGU General Assembly Conference Abstracts, 17: 10494

Nikolaidis R. 2002. Observation of geodetic and seismic deformation with the Global Positioning System. Dissertation for Doctoral Degree. San Diego: University of California

Park K D, Nerem R S, Davis J L, Schenewerk M S, Milne G A, Mitrovica J X. 2002. Investigation of glacial isostatic adjustment in the northeast U.S. using GPS measurements. Geophys Res Lett, 29: 1509

Paulson A, Zhong S, Wahr J. 2007. Inference of mantle viscosity from GRACE and relative sea level data. Geophys J Int, 171: 497-508

Peltier W R. 2004. Global glacial isostasy and the surface of the ice-age Earth: The ICE-5G (VM2) model and GRACE. Annu Rev Earth Planet Sci, 32: 111-149

Peltier W R, Argus D F, Drummond R. 2015. Space geodesy constrains ice age terminal deglaciation: The global ICE-6G_C (VM5a) model. J Geophys Res-Solid Earth, 120: 450-487

Prandi P, Cazenave A, Becker M. 2009. Is coastal mean sea level rising faster than the global mean? A comparison between tide gauges and satellite altimetry over 1993-2007. Geophys Res Lett, 36: L05602

Pritchard M. 2010. Deformation explained. Nat Geosci, 3: 515

Takada Y, Fukushima Y. 2013. Volcanic subsidence triggered by the 2011 Tohoku earthquake in Japan. Nat Geosci, 6: 637-641

Thomas I D, King M A, Bentley M J, Whitehouse P L, Penna N T, Williams S D P, Riva R E M, Lavallee D A, Clarke P J, King E C,
Hindmarsh R C A, Koivula H. 2011. Widespread low rates of Antarctic glacial isostatic adjustment revealed by GPS observations. Geophys Res Lett, 38: L22302

Wang H, Wu P. 2006. Effects of lateral variations in lithospheric thickness and mantle viscosity on glacially induced surface motion on a spherical, self-gravitating Maxwell Earth. Earth Planet Sci Lett, 244: $576-589$

Wang H, Wu P, van der Wal W. 2008. Using postglacial sea level, crustal velocities and gravity-rate-of-change to constrain the influence of thermal effects on mantle lateral heterogeneities. J Geodyn, 46: 104-117

Whitehouse P L, Bentley M J, Le Brocq A M. 2012a. A deglacial model for Antarctica: Geological constraints and glaciological modelling as a basis for a new model of Antarctic glacial isostatic adjustment. Quat Sci Rev, 32: 1-24

Whitehouse P L, Bentley M J, Milne G A, King M A, Thomas I D. 2012b. A new glacial isostatic adjustment model for Antarctica: Calibrated and tested using observations of relative sea-level change and present-day uplift rates. Geophys J Int, 190: 1464-1482

Wilson T J, Bevis M, Konfal S, Barletta V, Aster R, Chaput J, Heeszel D, Wiens D, Anandakrishnan S, Dalziel I, Huerta A, Kendrick E, Nyblade A, Winberry P, Smalley B, Lloyd A. 2015. Understanding glacial isostatic adjustment and ice mass change in Antarctica using integrated GPS and seismology observations. Vienna: EGU General Assembly Conference Abstracts. 17: 7762

Wu P, Wang H. 2006. Effects of mode coupling and location of rotational axis on glacial induced rotational deformation in a laterally heterogeneous viscoelastic earth. Geophys J Int, 167: 853-859

(责任编委: 李新) 